\title{
A single task assessment system of upper-limb motor function after stroke
}

\author{
Jiuwei Li ${ }^{\mathrm{a}}$, Bingyu Pan ${ }^{\mathrm{a}}$, Tingting Jin ${ }^{\mathrm{b}}$, Zhipei Huang ${ }^{\mathrm{a}, *}$, Shiwei $\mathrm{Ye}^{\mathrm{a}}$, Jiankang $\mathrm{Wu}^{\mathrm{a}}$, \\ Zhen Huang ${ }^{\mathrm{b}}$, Bin Xie ${ }^{\mathrm{b}}$, Chun Luo ${ }^{\mathrm{b}}$ and Cui Wang ${ }^{\mathrm{b}}$

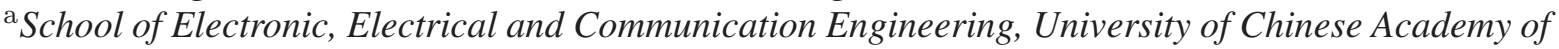 \\ Science, Beijing, China \\ ${ }^{\mathrm{b}}$ Rehabilitation Department, Peking University $1^{\text {st }}$ Hospital, Beijing, China
}

\begin{abstract}
.
BACKGROUND: Nowadays, stroke is a leading cause of disability in adults. Assessment of motor performance has played an important role in rehabilitation for post stroke patients. Therefore, it is quite important to develop an automatic assessment system of motor function.

OBJECTIVE: The purpose of this study is to assess the performance of the single task upper-limb movements quantitatively among stroke survivors.

METHODS: Eleven normal subjects and thirty-five subjects with stroke were involved in this study. The subjects, who were wearing the micro-sensor motion capture system, performed shoulder flexion in a sitting position. The system recorded threedimensional kinematics data of limb movements in quaternions. By extracting the significant features from these data, we built a linear model to acquire the functional assessment score (FAS).

RESULTS: All of the kinematics features have a significant statistical difference $(P<0.05)$ between patients and healthy people, while the feature values have a high correlation with Fugl-Meyer (FM) scores $(r>0.5, p<0.05)$, indicating that these features are able to reflect the level of motion impairment. Furthermore, most samples of the linear model locate in the confidence interval after regression, with the residual approaching a normal distribution. These results show that the FAS is capable of motor function assessment for stroke survivors.

CONCLUSION: These findings represent an important step towards a system that can be utilized for precise single task motor evaluation after stroke, applicable to clinical research and as a tool for rehabilitation.
\end{abstract}

Keywords: Stroke, rehabilitation, motor assessment, upper-limb, micro-sensor

\section{Introduction}

Stroke is a leading cause of disability in adults, and its incidence is expected to increase with the aging people [1]. Assessment of motor performance has played a very important role in Neuro-rehabilitation and research, especially for survivors early after stroke [2,3]. As the report shows, severe impairments of the upper-limb happen as much as 50\% among stroke survivors [4]. The outcomes of upper-limb rehabilitation lag behind those for the lower limb in ambulation. One of the major reasons is the lack of an efficient assessment technique.

\footnotetext{
${ }^{*}$ Corresponding author: Zhipei Huang, Sensor Networks and Applications Research Center, School of Electronic, Electrical and Communication Engineering, University of Chinese Academy of Science, Huaibei Town 380, Huairou District, Beijing, China. Tel.: +86 01069671882; E-mail: zhphuang@ucas.ac.cn.
}

0928-7329/16/\$35.00 (C) 2016 - IOS Press and the authors. All rights reserved This article is published online with Open Access and distributed under the terms of the Creative Commons Attribution NonCommercial License. 
In this context, researchers and clinicians have focused their attention on the development and application of the assessment to motor performance. In traditional, motor assessment is based on the clinicians' judgment, which depends on their acute observational skill entirely. Due to the diversity of the clinicians' expertise, it is hard to guarantee the consistency of assessment criteria. Two of the most acceptable approaches are Wolf Motor Function Test (WMFT) and Fugl-Meyer (FM) assessment method [5,6].

On the other hand, some researchers employ robot-aided rehabilitation facilities to record the quantitative movement data, which could provide useful information to evaluate motor performance without clinicians' experience [7,8]. While, the range and degree of freedom of motion are limited by the robotic machines [9], causing the evaluating results of functional motor performance inconsistent with our daily life ones. Another way to record patients' kinematic data is optical method by measuring the location of infrared reflective markers attached to the patient via several cameras [10,11]. These systems have no limits to the range or degree of freedom of motion, which make a minimal burden to the patients. However, both the robotic and optical systems require a lot financially and spatially, leading to their employment in rehabilitation center only.

Recently, wearable sensor system has a good momentum in this field. Patel used accelerometers to record the motion of stroke survivors. Through these data, they extracted some features and quantitatively evaluated the performance of the motion $[12,13]$. This study perfectly overcomes the drawbacks of traditional assessments, such as the waste of human resources and diverse results due to subjective evaluation. In addition, it is more inexpensive and portable than robotic and video systems. Nevertheless, it is obviously difficult to represent the motion completely and to extract significant features only by acceleration data. Cruz and his group developed a portable system instead of manual WMFT. They collected kinematics data by their motion capture system and quantitatively evaluate the motion performance based on the classified method according to WMFT [14]. However, they merely acquired the binary features in each task, which cannot provide sufficient information for further study in rehabilitation.

In this paper, we present a novel approach to assess upper-limb movements quantitatively by a single task. A Micro-Sensor system records the movements of people. Later, five quantized significant features are extracted using a new method. At last, a linear model is established to get a comprehensive evaluation result of the movement.

\section{Materials and methods}

\subsection{Subjects}

Thirty-five stroke survivors and eleven age-matched control subjects participated in the study, who were recruited from the Peking University first hospital. All patients studied suffered from a unilateral cortical and subcortical lesion resulting from either an ischemic or a hemorrhagic stroke. They were assessed functional impairment of the upper-limb by a Fugl-Meyer score less than 66. All participants gave informed consent before experimentations.

\subsection{Test description}

The subjects were requested to sit, wearing our micro-sensor motion capture system. After receiving the instruction, they performed shoulder flexion to 90 degrees and held on for 2-3 seconds, the same way as getting objects in our daily life. The system recorded real-time motion data throughout the whole experiment. 


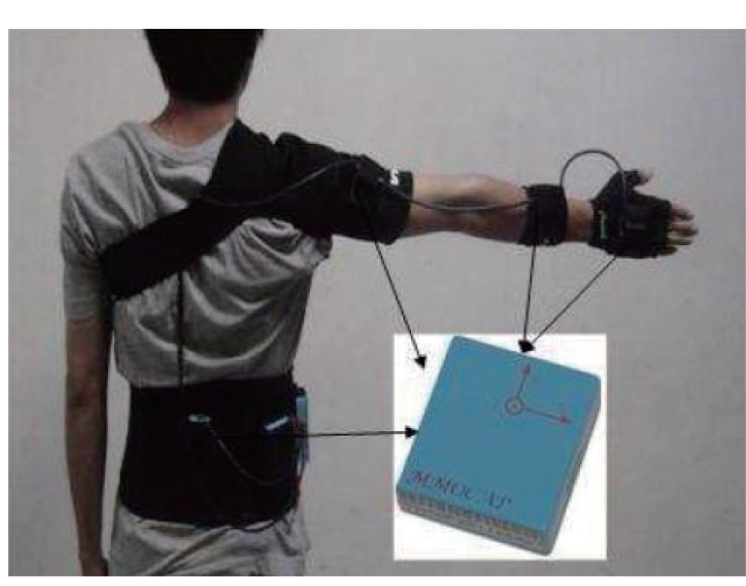

Fig. 1. The motion capture system.

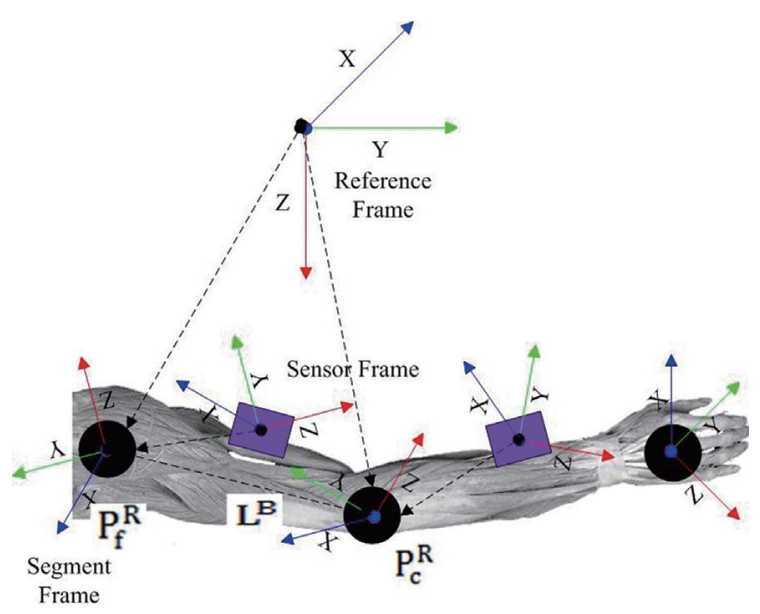

Fig. 2. Senor segment mapping and the hierarchical upper limb model.

\subsection{Motion capture system}

The motion capture system is a set of wearable wireless devices exploited by our team, as Fig. 1 . The system consists of several sensor modules to record the movement of human body. Each of sensor modules integrate 9-axis sensor that combines a 3-axis MEMS gyroscope, a 3-axis MEMS accelerometer, and a 3-axis MEMS magnetometer (sees MPU9150 datasheet for more details). We implement the quaternion-based MARG (Magnetic, Angular Rate, and Gravity) sensor fusion algorithm to estimate the orientation of the modules [15]. With the sensor modules fixed on the body segments, the human motions could be obtained by our kinematics algorithm on the basis of the orientation of sensors. The senor segment mapping and the hierarchical upper limb model is shown in Fig. 2. In this experiment, data from two modules, which are placed on torso and upper-arm, are involved to track shoulder activity.

\subsection{Data processing}

Quaternions are very useful and have been applied successfully in Vision and Graphics. A quaternion has the form of Eq. (1), which can be used to rotate any 3D point in space, around an arbitrary axis [16]. The trunk, upper-arm, forearm, and hand are regarded as rigid bodies. Therefore, the rotation of joint can be expressed in quaternion, computed by the orientation of conjoint limbs as Eq. (2). The symbol $q^{L_{1} L_{2}}$ represents the rotation of joint, and $q^{G L_{1}}, q^{G L_{2}}$ are the orientations of the limbs.

$$
\begin{aligned}
& q=\left(\begin{array}{llll}
q_{0} & q_{1} & q_{2} & q_{3}
\end{array}\right) \\
& q^{L_{1} L_{2}}=\left(q^{G L_{1}}\right)^{-1} \otimes q^{G L_{2}}
\end{aligned}
$$

In most of the studies, each limb segment corresponded to a translational 3D vector in a kinematic model. The length of each segment is specified according to the normalized dimensions. The current $3 \mathrm{D}$ position of each joint is calculated to represent upper-limb movement $[14,17,18]$. However, there are many defects in this way. Firstly, simplifying the rigid body to 3D vector may cause the loss of the degree of freedom (roll rotation). Secondly, for further data analysis, the trajectory of joint need to be projected into a plane of motion and a corresponding vertical plane. Since the motion plane is always 
defined by people, there would be a deviation from the actual plane, which can bias the results of the analysis.

In this paper, to identify the occurrence of movement of the shoulder joint, the real-time rotation angle $\theta$ and rotation axis $[x, y, z]$ are computed using quaternion from its initial to the moment as Eqs (3) and (4).

$$
\begin{aligned}
\theta & =2 * \arccos \left(q_{0}\right) \\
{[x, y, z] } & =\left[\frac{q_{1}}{\sin \frac{\theta}{2}}, \frac{q_{2}}{\sin \frac{\theta}{2}}, \frac{q_{3}}{\sin \frac{\theta}{2}}\right]
\end{aligned}
$$

\subsection{Feature extraction}

To exploit more information, significant features are extracted from the motion data. With these features, the performance of the motion can be explored better. Before feature extraction, the motion data is sectioned into two subsections: isometric contraction and isotonic contraction. Features are extracted from these parts respectively. There are five features given as follows.

\subsubsection{Torso balance}

In the clinical practice, some patients can hardly complete the action by controlling their upper limbs. Therefore, torso movement is involved as a compensation. To quantify the compensation of the torso, the change of attitude is recorded throughout the whole movement. The greater the value is, the more serious the patient's torso compensatory condition is. Torso balance is calculated as Eq. (5), in which $\theta_{T o r}$ is the rotation angle of the torso estimated via Eq. (3) during the whole movement.

$$
\mathrm{Bal}_{\text {Tor }}=\log \left[\operatorname{var}\left(\theta_{\text {Tor }}\right)\right]
$$

\subsubsection{Range of motion}

Each specific joint has a normal range of motion. A limited range of motion indicates that the joint has a reduction in its ability to move. The reduced motion may reflect a mechanical problem with the specific joint caused by stroke. The range of motion is a very useful indicator to quantify the performance of movement, and an important factor in most impairment guides as well $[19,20]$. In this paper, the mean value of joint angle during isometric contraction was defined in the range of motion as Eq. (6), in which $\theta_{\text {met }}$ is the rotation angle of the shoulder during isometric contraction calculated in Eq. (3).

$$
\operatorname{Ran}_{\text {Mot }}=\operatorname{mean}\left(\theta_{\text {met }}\right)
$$

\subsubsection{Angle divergence}

Stroke patients usually suffer from upper-limb motion disorders and cannot maintain a posture for a period of time. The ability to maintain a posture can be quantified by the change in the angle of the joints during isometric contraction, in the form of angle divergence as Eq. (7), where $a$ is the weight of the impact factor in angle, and $\left[x_{m e t}, y_{m e t}, z_{m e t}\right]$ is the rotation axis of the shoulder during isometric contraction obtained in Eq. (4).

$$
A n g_{D i v}=\log \left[a * \operatorname{var}\left(\theta_{m e t}\right)+\operatorname{var}\left(x_{m e t}\right)+\operatorname{var}\left(y_{m e t}\right)+\operatorname{var}\left(z_{m e t}\right)\right]
$$




\subsubsection{Movement velocity}

Stroke always causes reduction of movement efficiency, which is a prominent appearance of motor ability reduction. The movement velocity of the shoulder is expressed as Eq. (8). $\theta_{\text {start }}, \theta_{\text {end }}$ are initial and final rotation angle of the shoulder during isotonic contraction respectively acquired through Eq. (3); $t$ is the time duration of isotonic contraction.

$$
V e l_{\text {Mov }}=\frac{\theta_{\text {end }}-\theta_{\text {start }}}{t}
$$

\subsubsection{Smoothness of movement}

The smoothness of movement has a high correlation with motor ability. Most studies used jerk metric of the joint trajectory to quantify the smoothness of movement [21]. In this paper, we use the stability of the rotation axis to quantify the smoothness of movement, which has been proved better than jerk in this application, as Eq. (9). [ $x y z]$ is the axis of rotation during isotonic contraction calculated by Eq. (4); $x_{f i t}, y_{f i t}, z_{f i t}$ is the polynomial fitting curve of $x, y, z$ respectively, obtained by MATLAB function polyfit.

$$
\operatorname{Smo}_{\text {Mov }}=\log \left[\operatorname{mean}\left(x-x_{f i t}\right)+\operatorname{mean}\left(y-y_{f i t}\right)+\operatorname{mean}\left(z-z_{f i t}\right)\right]
$$

\subsubsection{Nonlinear transformation}

To make it reflect motor function intuitively and fit the model better, the features should have a high linear correlation with FM score. To improve the linear correlation, the function $\log$ is involved as a nonlinear transformation in this study, as Eqs (5), (7) and (9).

\subsubsection{Validity of the feature}

Two kinds of evaluation standards are applied to verify the validity of the features. Firstly, the features should distinguish between patient group and normal group. Statistical hypothesis testing is used to estimate significant difference. The test rejecting the null hypothesis at the 0.05 significance level is acceptable. Secondly, the feature and FM score should have the linear correlation. It is acceptable if the linear correlation coefficient is more than 0.5 and p-value is less than 0.05 . The results of the evaluation of the range of the motion are shown in Fig. 3, suggesting that all the features meet both of the requirements above.

\subsection{Function assessment score}

To get a comprehensive assessment result of the upper-limb motor function in the single task, the linear model of the features is established to calculate FAS, shown as Eq. (10). $X_{1}-X_{5}$ represent the values of the features; $\beta_{0}-\beta_{5}$ are the corresponding weight coefficients, which are obtained by optimizing. There is a consensus that FAS should have a correlation with FM score. Therefore, we apply multiple linear regression to determine the optimal coefficient.

$$
F A S=\beta_{0}+\beta_{1} X_{1}+\beta_{2} X_{2}+\beta_{3} X_{3}+\beta_{4} X_{4}+\beta_{5} X_{5}
$$


Table 1

\begin{tabular}{lrrr}
\multicolumn{3}{c}{ The mean and variance of feature value } \\
\cline { 2 - 3 } & \multicolumn{1}{c}{ Mean \pm Standard deviation } & $P(<0.05)$ \\
\cline { 2 - 3 } & \multicolumn{1}{c}{ Affect } & \multicolumn{1}{c}{ Control } & \\
\hline Bal $_{\text {Tor }}$ & $0.60 \pm 1.677$ & $-1.80 \pm 1.123$ & 0.000 \\
Ran $_{\text {Mot }}$ & $48.5 \pm 15.48$ & $67.8 \pm 7.387$ & 0.000 \\
Ang $_{\text {Div }}$ & $-8.35 \pm 1.519$ & $-10.5 \pm 0.623$ & 0.000 \\
Vel $_{\text {Mov }}$ & $1.39 \pm 0.442$ & $6.16 \pm 0.677$ & 0.000 \\
Smo $_{\text {Mov }}$ & $-4.43 \pm 0.658$ & $-4.98 \pm 0.282$ & 0.011 \\
\hline
\end{tabular}
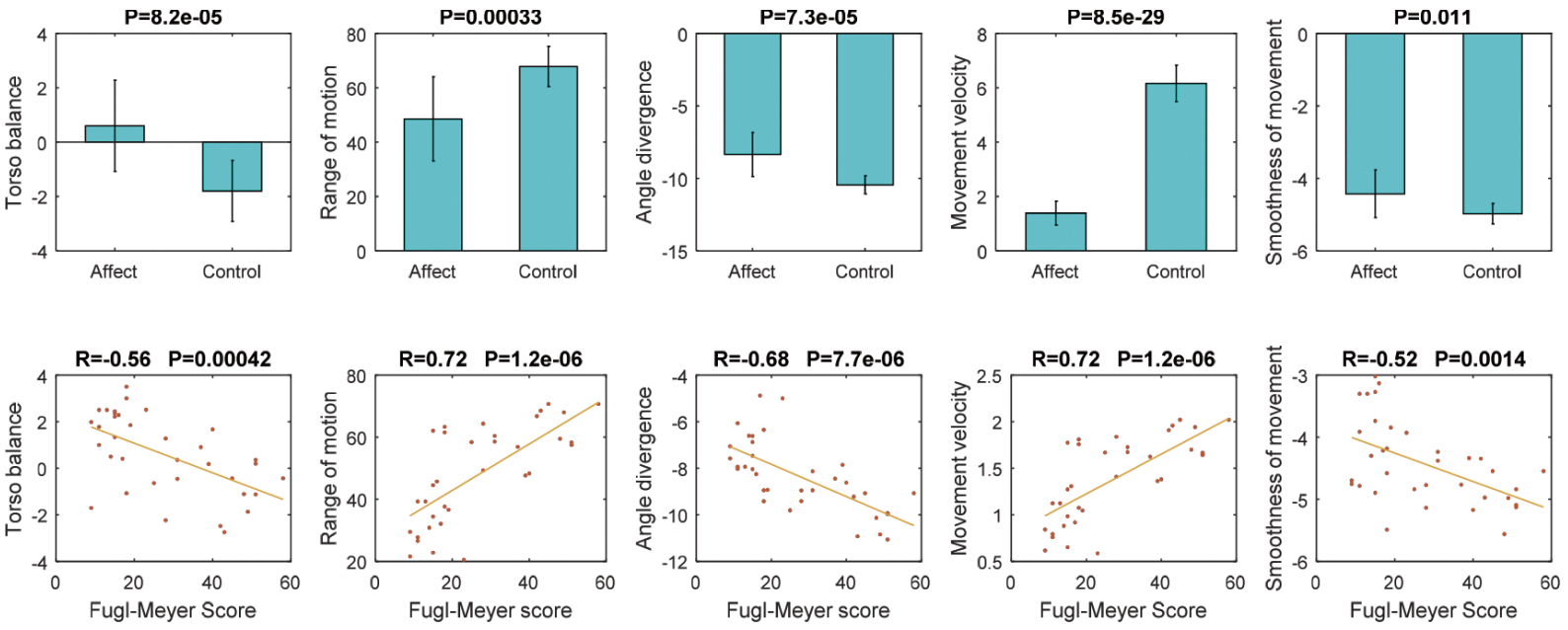

Fig. 3. Analysis of significant difference and correlation.

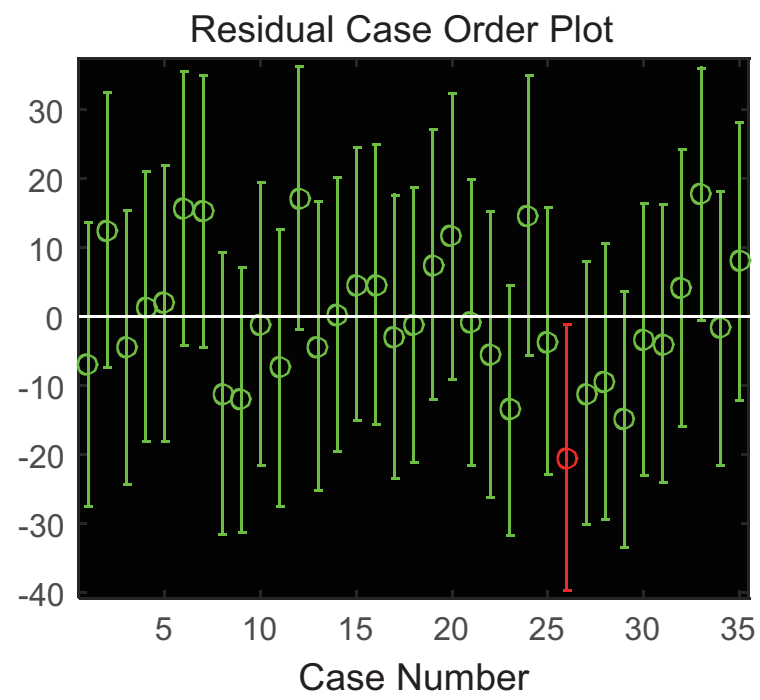

Fig. 4. Residual case order plot.

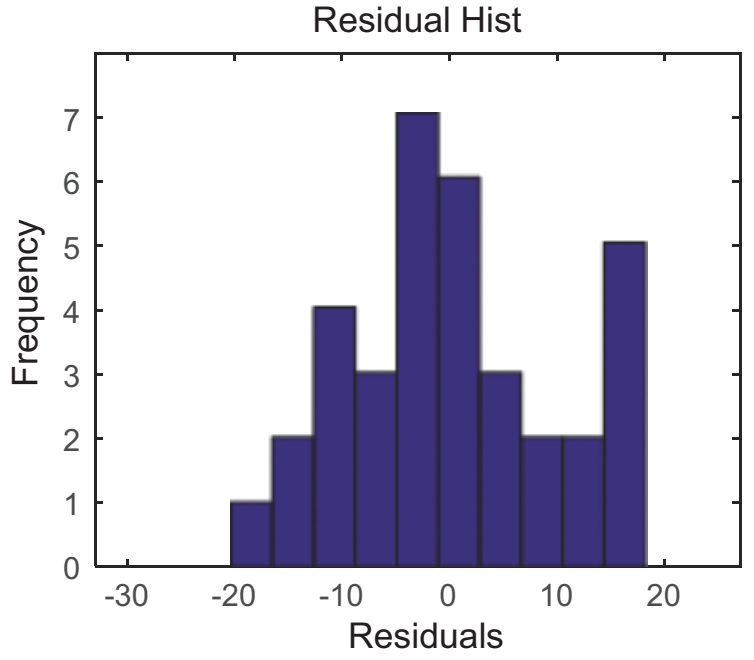

Fig. 5. Residual histogram. 
Table 2

The correlation between feature value and FM score

\begin{tabular}{lcc}
\hline Evaluation indicators & Related coefficient & $P(<0.05)$ \\
\hline Bal $_{\text {Tor }}$ & -0.56 & 0.000 \\
Ran $_{\text {Mot }}$ & 0.72 & 0.000 \\
Ang $_{\text {Div }}$ & -0.68 & 0.000 \\
Vel $_{\text {Mov }}$ & 0.72 & 0.000 \\
Smo $_{\text {Mov }}$ & -0.52 & 0.001 \\
\hline
\end{tabular}

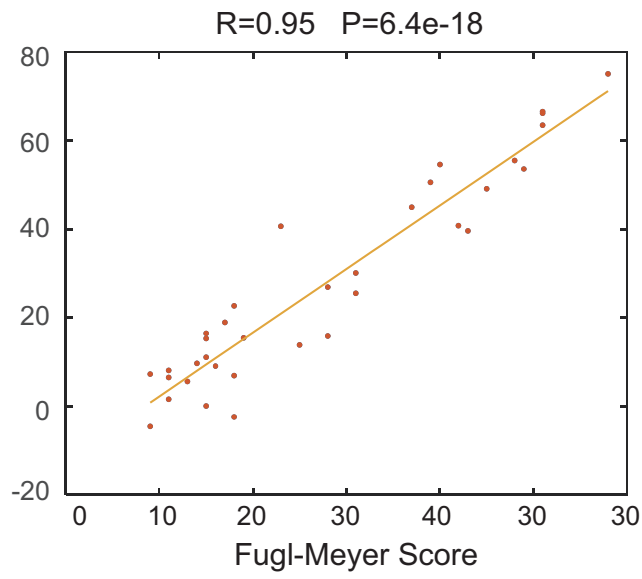

Fig. 6. The shoulder flexion FAS and the Fugl-Meyer Score.

\section{Results}

\subsection{Feature validity}

Five significant features are extracted from eleven normal subjects and thirty-five subjects with stroke. Table 1 contains the mean and variance of patients group and healthy group. The result of the significant test shows that five features proposed in this paper have a significant statistical difference $(P<0.05)$ between patients and healthy people. Table 2 displays the correlation between feature value and FM score. All of the related coefficients' absolute value is more than 0.5 . The result suggests that the features are able to reflect the level of motion impairment.

\subsection{Regression model}

The parameters of linear model are determined via regression analysis. We apply the residual analysis to examine the quality of the fitted model. The error bar of the confidence intervals on the residuals is shown in Fig. 4 and residual histogram in Fig. 5. After regression, most samples of the linear model are within the confidence interval. Furthermore, the distribution of the residual is very similar to Gaussian. These justify the rationality of fitted model.

\subsection{Shoulder flexion FAS}

The quantitatively single task function assessment has been implemented. The shoulder flexion FAS and corresponding Fugl-Meyer score of thirty-five subjects with stroke (as Fig. 6) suggest that the FAS has a high liner correlation with Fugl-Meyer score in this experiment. 


\section{Conclusions}

In this study, an upper-limb quantitative assessment based on single task is proposed. The micro-sensor system accurately records kinematic data with great convenience. Different from former clinical scaling and similar automated method [14], this approach can acquire an integrated quantitative evaluation results aimed at a particular action rather than a simple rating. In addition, the five significant features based on real-time rotation angle and axis reflect the performance of upper-limb movement intuitively and are very helpful for the diagnosis and rehabilitation of stroke.

The FAS, as an evaluation of a particular task proposed in this paper, is acquired via the linear model optimized by FM score. In the future, we may not need the new patient's FM score any more, if there were the enough dataset to support. The performance of the regression model shows that it is possible to predict comprehensive upper-limb evaluation results roughly using single task assessment in Figure 6. To achieve a similar result of the clinical scaling assessment, several specific movements would be involved in this model.

\section{Acknowledgments}

This work is supported by the National Natural Science Foundation of China (Grant No. 81272166 and 61431017).

\section{References}

[1] D. Lloyd-Jones, R. J. Adams, T. M. Brown, M. Carnethon, S. Dai, G. De Simone, T. B. Ferguson, E. Ford, K. Furie, C. Gillespie, A. Go, K. Greenlund, N. Haase, S. Hailpern, P. M. Ho, V. Howard, B. Kissela, S. Kittner, D. Lackland, L. Lisabeth, A. Marelli, M. M. McDermott, J. Meigs, D. Mozaffarian, M. Mussolino, G. Nichol, V. L. Roger, W. Rosamond, R. Sacco, P. Sorlie, R. Stafford, T. Thom, S. Wasserthiel-Smoller, N. D. Wong, and J. Wylie-Rosett, Executive summary: Heart disease and stroke statistics-2010 update: A report from the american heart association, Circulation, vol. 121, no. 7, pp. 46-215, 2010.

[2] C. Stinear, Prediction of recovery of motor function after stroke, Lancet. Neurol., vol. 9, no. 12, pp. 1228-32, Dec. 2010.

[3] J. C. Hobart, S. J. Cano, J. P. Zajicek, and A. J. Thompson, Rating scales as outcome measures for clinical trials in neurology: problems, solutions, and recommendations, Lancet. Neurol., vol. 6, no. 12, pp. 1094-105, Dec. 2007.

[4] A. Heller, D. T. Wade, V. A. Wood, A. Sunderland, R. L. Hewer, and E. Ward, Arm function after stroke: measurement and recovery over the first three months, J. Neurol. Neurosurg. Psychiatry, vol. 50, no. 6, pp. 714-719, 1987.

[5] S. L. Wolf, P. A. Thompson, D. M. Morris, D. K. Rose, C. J. Winstein, E. Taub, C. Giuliani, and S. L. Pearson, The EXCITE trial: attributes of the Wolf Motor Function Test in patients with subacute stroke, Neurorehabil. Neural Repair, vol. 19, no. 3, pp. 194-205, 2005.

[6] Steglind S, Fugl-Meyer AR, Jääskö L, Leyman I, Olsson S, The post-stroke hemiplegic patient. 1. a method for evaluation of physical performance, Scand. J. Rehabil. Med., vol. 7(1): 13-31, 1975.

[7] P. S. Lum, C. G. Burgar, and H. F. M. Van der Loos, Quantification of force abnormalities during passive and activeassisted upper-limb reaching movements in post-stroke hemiparesis, IEEE Trans. Biomed. Eng., vol. 46, no. 6, pp. 652662, 1999.

[8] R. Colombo, I. Sterpi, A. Mazzone, C. Delconte, G. Minuco, and F. Pisano, Measuring changes of movement dynamics during robot-aided neurorehabilitation of stroke patients, IEEE Trans. Neural Syst. Rehabil. Eng., vol. 18, no. 1, pp. 75-85, 2010.

[9] A. Aa. Timmermans, H. Am. Seelen, R. D. Willmann, and H. Kingma, Technology-assisted training of arm-hand skills in stroke: concepts on reacquisition of motor control and therapist guidelines for rehabilitation technology design, $J$. Neuroeng. Rehabil., vol. 6, no. figure 1, p. 1, 2009.

[10] N. Yang, M. Zhang, C. Huang, and D. Jin, Motion quality evaluation of upper limb target-reaching movements, Med. Eng. Phys., vol. 24, pp. 115-120, 2002.

[11] A. J. G. Churchill, P. W. Halligan, and D. T. Wade, RIVCAM: A simple video-based kinematic analysis for clinical disorders of gait, Comput. Methods Programs Biomed., vol. 69, no. 3, pp. 197-209, 2002. 
[12] S. Patel, R. Hughes, T. Hester, J. Stein, M. Akay, J. G. Dy, and P. Bonato, A novel approach to monitor rehabilitation outcomes in stroke survivors using wearable technology, Proc. IEEE, vol. 98, no. 3, pp. 450-461, 2010.

[13] S. Patel, R. Hughes, T. Hester, J. Stein, M. Akay, J. Dy, and P. Bonato, Tracking motor recovery in stroke survivors undergoing rehabilitation using wearable technology, Conf. Proc. IEEE Eng. Med. Biol. Soc., vol. 2010, pp. 6858-6861, 2010.

[14] V. Tedim, V. Ferro, D. Dieteren, I. Araújo, C. Aguiar, and P. Coutinho, Medical Engineering \& Physics Technical note A novel system for automatic classification of upper limb motor function after stroke? An exploratory study, Med. Eng. Phys., vol. 36, no. 12, pp. 1704-1710, 2014.

[15] X. Y. X. Yun, C. Aparicio, E. R. Bachmann, and R. B. McGhee, Design, Implementation, and Experimental Results of a Quaternion-Based Kalman Filter for Human Body Motion Tracking, IEEE Trans. Robot., vol. 22, no. April, pp. 1216-1227, 2006.

[16] W. Paper, W. Paper, O. Matrices, and O. Matrices, Application of quaternions to computation with rotations, vol. 1, 1995.

[17] Huang Shuai, Luo Chun, Ye Shiwei, Liu Fei, Xie Bin, Wang Caifeng, Yang Li, Huang Zhen, Wu Jiankang, Motor impairment evaluation for upper limb in stroke patients on the basis of a microsensor, Int. J. Rehabil. Res., vol. 35, pp. 161-169, 2012.

[18] C. Wang, G. Tao, Z. Huang, J. Wu, F. Liu, S. Huang, and Z. Huang, Evaluation of Upper Limb Motion Function for Stroke Patients Rehabilitation, no. 60932001.

[19] R. L. Gajdosik and R. W. Bohannon, Clinical measurement of range of motion. Review of goniometry emphasizing reliability and validity, Phys. Ther., vol. 67, no. 12, pp. 1867-1872, 1987.

[20] J. W. Youdas, J. R. Carey, and T. R. Garrett, Reliability of measurements of cervical spine range of motion - comparison of three methods, Phys. Ther., vol. 71, no. 2, pp. 98-104; discussion 105-106, 1991.

[21] B. Rohrer, S. Fasoli, H. I. Krebs, R. Hughes, B. Volpe, W. R. Frontera, J. Stein, and N. Hogan, Movement smoothness changes during stroke recovery, The Journal of neuroscience: the official journal of the Society for Neuroscience, vol. 22, no. 18, pp. 8297-8304, 2002. 